\title{
A head and neck squamous cell carcinoma model to study and target HPV replication and transcription
}

\author{
Michael R. Evans ${ }^{1}$, Christian T. Fontan ${ }^{1}$, Claire D. James ${ }^{1}$, Xu Wang', Iain M. Morgan ${ }^{1,2}$, and \\ Molly L. Bristol1,* \\ 1 VCU Philips Institute for Oral Health Research, Department of Oral and Craniofacial Molecular Biology, \\ Virginia Commonwealth University School of Dentistry, Richmond, VA 23298, USA \\ 2 VCU Massey Cancer Center, Richmond, VA 23298, USA \\ * Correspondence: mlbristol@vcu.edu; Tel.: +1-804-828-5702
}

\begin{abstract}
The incidence of human papillomavirus-related head and neck squamous cell carcinoma (HPV+HNSCC) has reached epidemic levels in the last decade. While prophylactic vaccines will prevent future HPV infections, there are currently no HPV-specific antiviral drugs to treat current HPV infections or HPV+HNSCC. HPV replication and transcription are promising targets for anti-HPV therapeutics, as modulation of these processes can alter expression levels of HPV E6 and E7, which are required for maintenance of the transformed phenotype. This is a particularly attractive target in in HPV+HNSCC where the majority of tumors have episomal genomes replicating in an E1-E2 dependent manner. Here, we describe a model system to study HPV16 E1-E2 mediated DNA replication and HPV16 E2-mediated transcriptional activation and repression in multiple HNSCC cell lines. Our results demonstrate that low levels of IFIT1 are required for HPV16 replication in HNSCC cell lines and HPV16 E1 interacts with IFIT1. Restoration of IFIT1 expression in HNSCC cell lines partially inhibits HPV16 E1-E2 mediated replication. This system can be used to study replication and transcription by HPV16 E1 and E2 in HNSCC as well as be utilized to screen potential anti-HPV therapeutics that target HPV16 replication and transcription.
\end{abstract}

Keywords: papillomaviruses; HPV; replication; transcription; E1; E2; IFIT1; cancer; HNSCC

\section{Introduction}

Human papillomaviruses (HPVs) are double-stranded DNA viruses that cause a variety of diseases ranging from warts to cancer [1]. HPV is the most common sexually transmitted infection in the United States, with an estimated 80 percent of sexually active adults acquiring a HPV-related infection in their lifetime [2]. Of the high-risk HPVs known to be causative in the development of cancer, HPV16 is the most prevalent genotype [3]. HPV16 is causative in around 50\% of cervical cancers and nearly $90 \%$ of HPV-related head and neck squamous cell carcinomas (HPV+HNSCCs) $[1,3]$. The prevalence of HPV+ HNSCCs has been rapidly increasing in the last decade, with over half a million new cases per year worldwide [4]. While prophylactic vaccines are available that will reduce future HPV16 disease burden by preventing future HPV16 infections, current vaccines are not therapeutic and do not benefit patients with current HPV infections. Additionally, overall vaccine coverage is low, with less than half of American adolescents receiving all of the recommended doses of HPV vaccines [5]. Further understanding of HPV16 and its life cycle in the head and neck region is needed in order to develop novel anti-viral therapies against HPV16.

In HPV infected cells, the E6 and E7 oncoproteins degrade p53 and pRb leading to cellular proliferation, prevention of apoptosis and, over time, cellular transformation [6-14]. Reducing E6 
and E7 levels induces apoptosis or senescence of HPV-transformed cells via restoration of p53 and $\mathrm{pRb}$, making these viral oncoproteins a viable target for anti-HPV therapeutics [15-22]. One way to reduce E6 and E7 levels, particularly in $\mathrm{HPV}+\mathrm{HNSCC}$, is targeting viral replication. Contrary to $\mathrm{HPV}+$ cervical cancers, recent evidence suggests that the HPV genome remains episomal in a majority of HPV+ HNSCCs, and is replicated in a E1-E2 dependent manner [23-26]. Reducing E1-E2 dependent replication in HPV+HNSCC would reduce viral genome levels, thereby reducing transcript levels and subsequently the protein levels of E6 and E7. To our knowledge, no characterized system exists to study HPV16 E1-E2 mediated replication in HNSCC. In addition to targeting replication, directly targeting transcription of E6 and E7 is also a viable approach to reducing the levels of these oncoproteins in HPV+HNSCC. Numerous studies have shown that adding exogenous E2 (a negative regulator of E6/E7 transcription when expressed at high levels) to HPV-transformed cervical cancer cells leads to their apoptosis or senescence, presumably due to a decrease in E6 and E7 levels [27-31]. This is supported by work demonstrating that silencing E6 or E7 in HPV-transformed cervical cancer cell lines leads to similar effects [15-18, 20]. Further investigation of HPV16 transcription in HNSCC is needed to determine the efficacy of similar approaches in HNSCC as well to determine potential novel strategies for targeting E6 and E7 transcription in these cancers.

Here, we describe a model system to investigate HPV16 E2-mediated transcription and HPV16 E1-E2 mediated DNA replication in multiple HNSCC cell lines. We observe E2-dependent transcriptional activation and repression in HN-22 and HN-31 HNSCC cell lines, the pattern of which may be unique to HNSCC. We also observe detectable, reproducible amounts of HPV16 E1-E2 mediated replication in these lines. Performing the E1-E2 mediated replication assay in multiple HNSCC cell lines, we discovered that high levels of endogenous interferon induced protein with tetratricopeptide repeats 1 (IFIT1) prevents measurable E1-E2 DNA replication (observed in nu61, SCC-61, HN-11, and HN-30). Adding exogenous IFIT1 to HN-22 (no detectable endogenous IFIT1) and performing immunoprecipitation experiments revealed an interaction between IFIT1 and HPV16 E1. This E1-IFIT1 interaction reduced E1-E2 mediated replication. Overall, this model system can be used to study HPV16 E1-E2 mediated replication and E2-dependent transcription in HNSCC cells and could be used as a tool to screen replication and transcription targeting anti-HPV agents.

\section{Materials and Methods}

\subsection{HNSCC cell lines and cell culture}

The HN-4, HN-8, HN-11, HN-22, HN-30, and HN-31 cell lines have been described previously [32]. SCC-61 and radioresistant nu61 cell lines have also been previously described [33]. All cell lines were grown in Dulbecco's Modified Eagle's Medium (Invitrogen) supplemented with 10\% fetal bovine serum (VWR) and passaged every 3 days. All cell lines were routinely tested for the presence of mycoplasma.

\subsection{Plasmids}

All plasmids utilized in this study have been previously described: HPV16 pOri (pOri) [34], HPV16 E1-HA (hemagglutinin, HA) (E1) [35], HPV16 E2 (E2) [36], pcDNA3.1 3xFlag IFIT1 (IFIT1) [37], pcDNA3.1 (Invitrogen), pLX302 [38], pBABE-hygro [39], pGL3 [34], pTK6E2 [40]. pGL3-16LCR was generated by PCR amplification of the HPV16 LCR from W12 cells, introducing KpnI and BglIII restriction sites, and cloned into a pGL3 backbone. 


\section{6}

\subsection{Transient E1-E2 mediated DNA replication assay}

Transient E1-E2 mediated DNA replication assays were performed as described previously [34,41,42]. Briefly, $5 \times 10^{5}$ HNSCC cells were plated onto $100 \mathrm{~mm}^{2}$ cell culture dishes. The next day, plasmid DNA was transfected into HNSCC cells using the calcium phosphate method [43]. Three days after transfection, low molecular weight DNA was extracted using the HIRT method as described previously [42]. Sample DNA was extracted twice with phenol:chloroform:isoamyl alchohol (25:24:1) and precipitated with ethanol. Samples were then centrifuged, and the resulting DNA pellet was washed with $70 \%$ ethanol, dried, and resuspended in $100 \mu \mathrm{L}$ of nuclease free water. $30 \mu \mathrm{L}$ of sample DNA were digested with DpnI (New England Biolabs) overnight to remove input HPV16 pOri or with MboI (New England Biolabs) to remove replicated HPV16 pOri. Quantity of replicated HPV16 pOri DNA relative to input was determined by real-time PCR as described previously [34].

\subsection{E2 transcriptional activation and repression assays}

HN-22 and HN-31 cells were plated at $5 \times 10^{5}$ in $100-\mathrm{mm}$ dishes. The following day, the calcium phosphate method [43] was used to transfect cells with 1 $1 \mu \mathrm{g}$ pGL3 basic, pTK6E2 or pGL3-16LCR plasmids and varying concentrations of HPV16 E2 plasmid (1-1000ng). 24-hours post transfection cells were washed and media replaced. 48-hours post transfection, cells were harvested utilizing Promega Reporter Lysis Buffer and analyzed for luciferase using the Promega Luciferase Assay System. Concentrations were normalized to protein levels, as measured by the BioRad Protein Assay Dye, and relative luciferase units were measured using the BioTek Synergy H1 Hybrid Reader.

\subsection{Western blots}

HNSCC cells were transfected using calcium phosphate method [43]. Cells were harvested with trypsin, washed with PBS, and resuspended in 50 $\mathrm{L}$ lysis buffer $(0.5 \%$ Nonidet P-40, 50mM Tris- $\mathrm{HCl}$ $\mathrm{pH} 8,150 \mathrm{mM} \mathrm{NaCl}$ ) supplemented with protease inhibitor (Roche Molecular Biochemicals) and $0.1 \%$ phosphatase inhibitor cocktail (Sigma). Cells were left in lysis buffer mixture on ice for 30 minutes, centrifuged at $18,000 \mathrm{RCF}$ at $4^{\circ} \mathrm{C}$, and supernatant was collected. Total protein levels were determined utilizing the Bio-Rad protein estimation assay and equal amounts of protein were then boiled in 2x Laemmli sample buffer (Bio-Rad). Samples were loaded onto a Novex 4-12\% gradient Tris-Glycine gel (Invitrogen), run at 100V, and transferred onto nitrocellulose membranes (Bio-Rad) via the wet blot method overnight at 30V. Membranes were blocked in Odyssey blocking buffer (LI-COR) diluted 1:1 in PBS and probed with relevant antibody diluted in the same blocking buffer overnight at $4^{\circ} \mathrm{C}$. Membranes were then washed with PBS with $0.1 \%$ Tween (PBS-Tween) and probed with Odyssey secondary antibody (goat anti-mouse IRdye800cw or goat anti-rabbit IRdye680cw, LI-COR) diluted 1:10,000 for 1 hour at $4^{\circ} \mathrm{C}$. Membranes were then washed with PBS-Tween before infrared scanning with the Odyssey CLx LI-COR imaging system. The following antibodies were used at the indicated concentrations: E1(HA) rabbit (1:1000, Abcam, ab9110), E2-TVG261 mouse (1:1000, Abcam, ab17185), IFIT1 rabbit (1:1000, Cell Signaling Technologies, D2X9Z), $\beta$-actin mouse (1:1000, Santa Cruz Biotechnology, sc-81178), $\beta$-actin rabbit (1:1000, Santa Cruz Biotechnology, sc-130656) GAPDH (1:2000, Santa Cruz Biotechnology, sc-47724). 
129

130

131

132

133

134

135

136

137

138

139

140

141

142

143

144

145

146

147

148

149

150

151

152

153

154

155

156

157

158

159

160

161

162

163

164

165

166

167

168

\subsection{Immunofluorescence}

HN-22 and HN-31 cells were grown on coverslips to $40 \%$ confluency and transfected with $1 \mu \mathrm{g}$ of the corresponding plasmids, listed in 2.2, using Lipofectamine 2000 (Life Technologies). 48 hours post-transfection, cells were fixed with chilled methanol and incubated at $-20^{\circ} \mathrm{C}$ for 5 minutes and then washed 3 times with PBS. Cells were then permeabilized with $0.2 \%$ Triton X-100 in PBS for 15 minutes and washed with PBS. Coverslips were blocked with 10\% normal goat serum (Life Technologies) for 1 hour then incubated with the following primary antibodies diluted in $10 \%$ normal goat serum overnight at $4^{\circ} \mathrm{C}$ : E1(HA) rabbit (1:1000, Abcam, ab9110), E2 mouse (unpurified TVG261, 1:20). Coverslips were then washed with PBS and incubated for 1 hour with a 1:1000 dilution of AlexaFluor 488 goat anti-rabbit secondary and AlexaFluor 594 goat anti-mouse secondary (Life Technologies). Coverslips were washed with PBS and cellular DNA was stained with $0.1 \mu \mathrm{g} / \mathrm{mL}$ DAPI (Santa Cruz sc-3598) for 15 minutes. Coverslips were again washed with PBS and mounted to using Vectashield medium (ThermoFisher) and sealed. Images were acquired using a Zeiss LSM 700 confocal laser scanning microscope configured around an AxioImager (Carl Zeiss). Images were analyzed using Zen lite software and Adobe Photoshop.

\subsection{Immunoprecipitation}

HN-22 cells were transfected using the calcium phosphate method [43] with $1 \mu \mathrm{g}$ of varying combinations of the plasmids listed in 2.2. Cells were harvested with trypsin and lysed according to the protocol described for western blotting in 2.4. $250 \mu \mathrm{g}$ of protein lysate was taken for each transfection condition and additional lysis buffer was added so that all samples totaled 500 $\mu \mathrm{L}$. $2 \mu \mathrm{L}$ of DYKDDDDK(FLAG) tag rabbit antibody (Invitrogen, PA1-984B) was added to each sample and samples were then rotated overnight at $4^{\circ} \mathrm{C}$. The next day, Protein A-Sepharose $4 \mathrm{~B}$ Fast Flow beads (Sigma) were washed five times with lysis buffer and then equilibrated with lysis buffer so that an equal volume of beads could be added to each sample. The lysate-bead mixture was rotated at $4^{\circ} \mathrm{C}$ for 6 hours and beads were washed five more times with lysis buffer to reduce nonspecific binding. Beads were then boiled in $4 x$ Laemmli sample buffer (Bio-Rad) and the supernatant of this mixture was run on a gel, transferred to a membrane, and visualized as described in section 2.5.

\section{Results}

3.1 Characteristics of the head and neck cancer cell lines used in this study

The HNSCC cell lines used in this study varied in tissue of origin, sex of patient, and p53 status. (Table 1). In order to determine transfectability of the selected cell lines, $1 \mu \mathrm{g}$ YFP was transfected via the calcium phosphate method. Ability to transfect was determined as greater than $5 \%$ of cells with visible YFP, although all cell lines deemed transfectable demonstrated $>50 \%$ transfection efficiency. (not shown). 
169

170

\begin{tabular}{|c|c|c|c|c|}
\hline Cell line & Sex of patient & Tissue of origin & p53 status & Ability to transfect \\
\hline nu61*1 & male & upper aerodigestive tract & deleted & yes \\
\hline SCC-61* & male & upper aerodigestive tract & deleted & yes \\
\hline $\mathrm{HN}-8^{n}$ & male & lymph node & deleted & no \\
\hline HN-11 & - & - & wild-type & yes \\
\hline $\mathrm{HN}-22^{z}$ & male & epiglottis & deleted & yes \\
\hline $\mathrm{HN}-31^{\wedge}$ & male & lymph node & mutated & yes \\
\hline
\end{tabular}

\footnotetext{
${ }^{1} \mathrm{SCC}-61$ cells made radioresistant in nude mice
}

Table 1. Head and neck squamous cell carcinoma lines utilized. Matching symbols next to cell line names indicate lines isolated from the same patient.

\subsection{HN-22 and HN-31 HNSCC cell lines allow E1-E2 mediated DNA replication}

Our lab has previously described a real-time PCR-based assay to detect transient E1-E2 mediated DNA replication that is more sensitive, less labor intensive, and less hazardous than southern blotting while also being highly quantitative [34]. This assay has primarily been performed in non-HNSCC cell lines such as c33a and 293T. In order to create a robust system to study and target HPV16 E1-E2 mediated DNA replication in HNSCC, we transfected nu61, SCC-61, HN-11, HN-22, $\mathrm{HN}-30$, and HN-31 with varying combinations of the components of the HPV16 replication complex (pOri, E1, E2). Nu61, SCC-61, HN-11, and HN-30 did not allow for measurable amounts of E1-E2 mediated DNA replication in cells (not shown). Conversely HN-22 and HN-31 lines allowed for quantifiable E1-E2 mediated DNA replication (Figure 1 A-C). 72 hours post-transfection with pOri, $\mathrm{E} 1$ and $\mathrm{E} 2 \mathrm{HN}-22$ and $\mathrm{HN}-31$ lines expressed appreciable amounts of the E1 and E2 proteins detectable by western blot (Figure $1 \mathrm{~A}$ ). HN-22 and HN-31 cells cotransfected with the complete HPV16 replication complex (pOri+ E1+E2) demonstrated a detectable DNA replication signal, while untransfected control cells (Ctl), or cells transfected with pOri or pOri+E1 demonstrated little to no DNA replication signal (Figure 1 B). Previous studies have demonstrated that E1 and E2 form distinct nuclear replication foci in the presence of the viral origin of replication $[41,44]$. To determine if this was the case in HNSCC cells, HN-22 and HN-31 were grown on coverslips and transfected with pOri+E1+E2. Coverslips were fixed and stained for E1(HA) and E2. As expected, E1 and E2 formed distinct replication foci in both HN-22 and HN-31 cells only when transfected with the complete replication complex (pOri+E1+E2) (Figure 1 C). These replication foci did not form unless cells were transfected with the complete replication complex. HN-22 cells transfected with pOri+E1 display diffuse nuclear staining for E1 (Figure 1 D). Overall, the results in Figure 1 demonstrate that we have created a system to consistently observe and quantify E1-E2 mediated DNA replication in the HNSCC lines HN-22 and HN-31. 
A

B
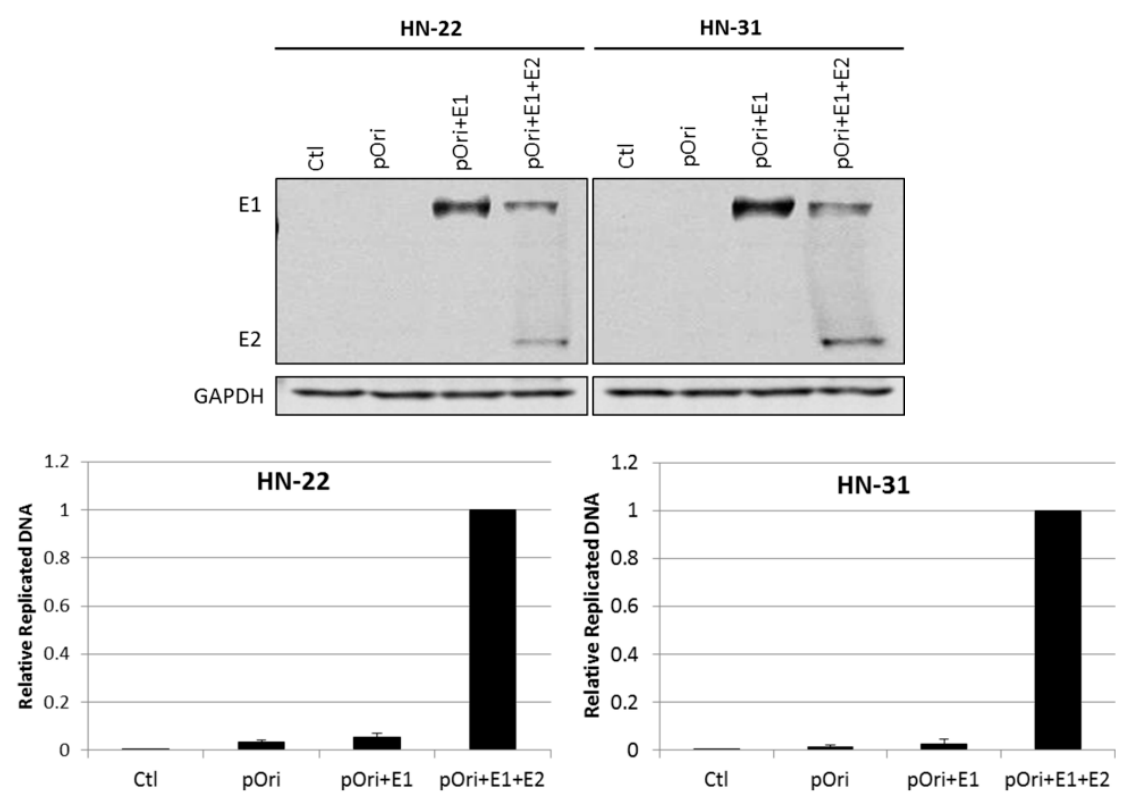

C
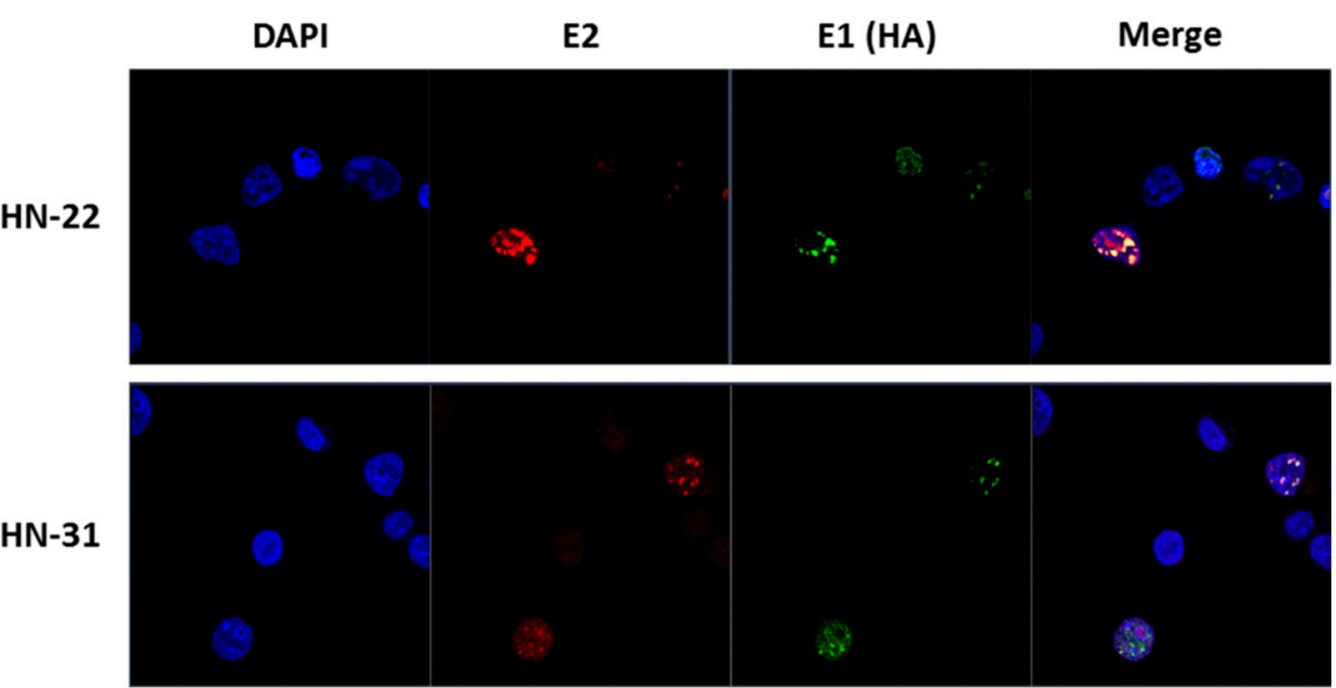

D

HN-31

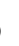

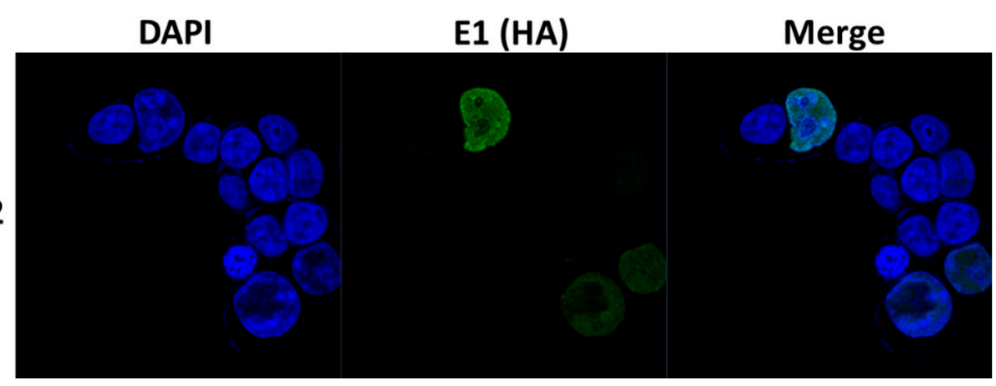

Figure 1. Characterization of E1-E2 mediated replication in HN-22 and HN-31 HNSCC cell lines. (A) Western blot analysis demonstrating expression of E1 and E2 after transfection of HN-22 and HN-31. GAPDH is shown as a loading control. (B) HN-22 and HN-31 cells were transfected with varying combinations of $1 \mu \mathrm{g}$ pOri, $1 \mu \mathrm{g}$ E1 and 10ng E2 and E1-E2 mediated replication was determined by real-time PCR for pOri. Significant replication was observed only in cells transfected with the full HPV16 replication complex (pOri+E1+E2) $(n=3$, error bars represent SEM). (C) Representative images of localization of E1 and E2 into nuclear replication foci in HN-22 and HN-33 cells transfected with pOri+E1+E2. (D) Replication foci are not present in HN-22 cells when transfected with pOri+E1 alone. Diffuse nuclear staining for E1 occurs in HN-22 transfected with pOri+E1. 
209

210

211

212

213

214

215

216

217

218

219

220

221

222

223

224

225

226

227

228

229

230

231

232

233

234

235

236

237

238

239

240

241

242

243

244

245

246

3.3 HN-22 and HN-31 HNSCC cell lines allow for E2-mediated transcriptional activation and repression

Because HN-22 and HN-31 lines allowed reproducible E1-E2 mediated replication, these lines were selected for study of E2-mediated transcription. To study transcriptional activation by E2, HN-22 and HN-31 cells were transfected with $1 \mu \mathrm{g}$ pTK6E2 and varying amounts of E2. pTK6E2 contains a thymidine kinase promoter with 6 HPV E2 binding sites which allow transactivation of downstream luciferase reporter upon binding of E2. pGL3, which has no E2 binding sites, serves as a negative control. Figure 2 A summarizes the results of the transcriptional activation assay in HN-22 and HN-31. HN-22 or HN-31 cells transfected with pGL3 basic or pTK6E2 alone demonstrated low, background levels of luciferase signal. Addition of 1ng of HPV16 E2 did little to change this basal level of signal. Upon addition of 10ng E2, a substantial increase in luciferase signal was measured in both HN-22 and HN-31 and this signal increased stepwise upon addition of 100ng or 1000ng E2. These results clearly demonstrate that E2 activates transcription from pTK6E2 in a dose dependent manner, signifying that E2-mediated transcription is occurring in HN-22 and HN-31 HNSCC cell lines

Perhaps more relevant to the HPV life cycle is the ability of E2 to repress transcription, particularly the transcription of E6 and E7. E2 transcriptionally represses E6 and E7 expression by binding to E2-binding sites in the LCR region of the HPV genome, thereby sterically hindering the action of cellular transcription factors on the HPV p97 early promoter and other proximal elements [45-49]. To study transcriptional repression by E2, $\mathrm{HN}-22$ and $\mathrm{HN}-31$ cells were transfected with $1 \mu \mathrm{g}$ pGL3-16LCR and varying amounts of E2. pGL3-16LCR contains the HPV16 LCR region from W12 cells upstream of a luciferase reporter. pGL3 serves as a negative control. Figure 2B demonstrates that there is low, background luciferase signal when HN-22 or HN-31 are transfected with pGL3, but measurable transcription is observed in cells transfected with pGL3-16LCR. Addition of 1ng of E2 does little to reduce the transcription of pGL3-16LCR. However, addition of 10ng E2 appears to reduce transcription by about $30 \%$ and addition of 100ng E2 appears to reduce transcription by $40 \%$. Interestingly, when 1000ng E2 is added an increase in transcription is observed in both HN-22 and HN-31. This is distinct from cervical cancer lines where E2 is a strong transcriptional repressor on HPV LCRs and remains so at high levels of E2 expression [50]. This may represent novel regulation of HPV E2-mediated transcriptional repression in HNSCC and this is currently being investigated in our lab. 
248
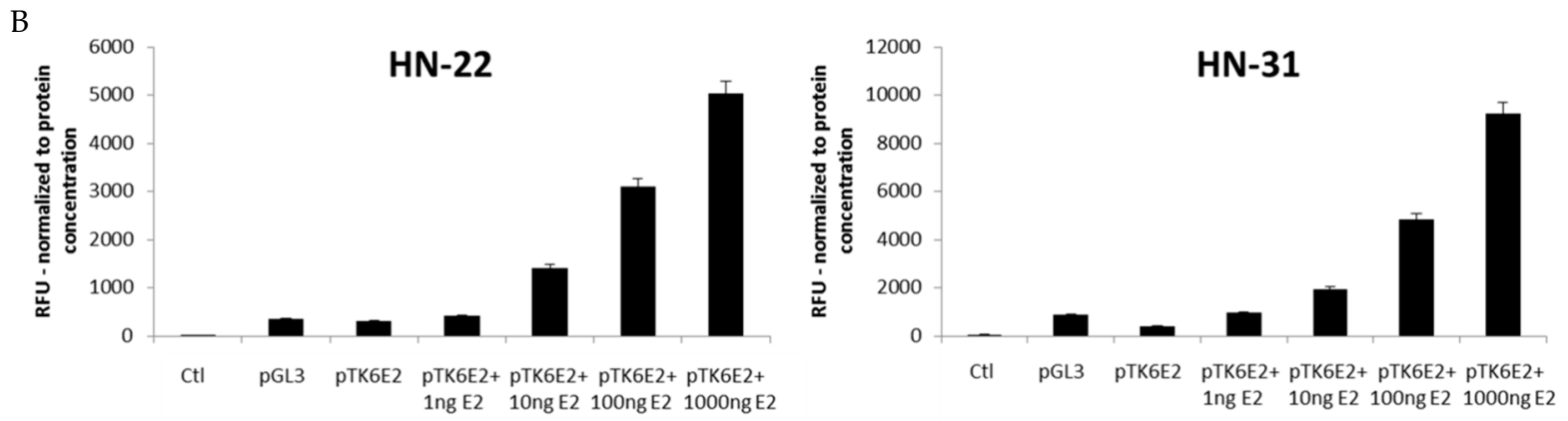

B
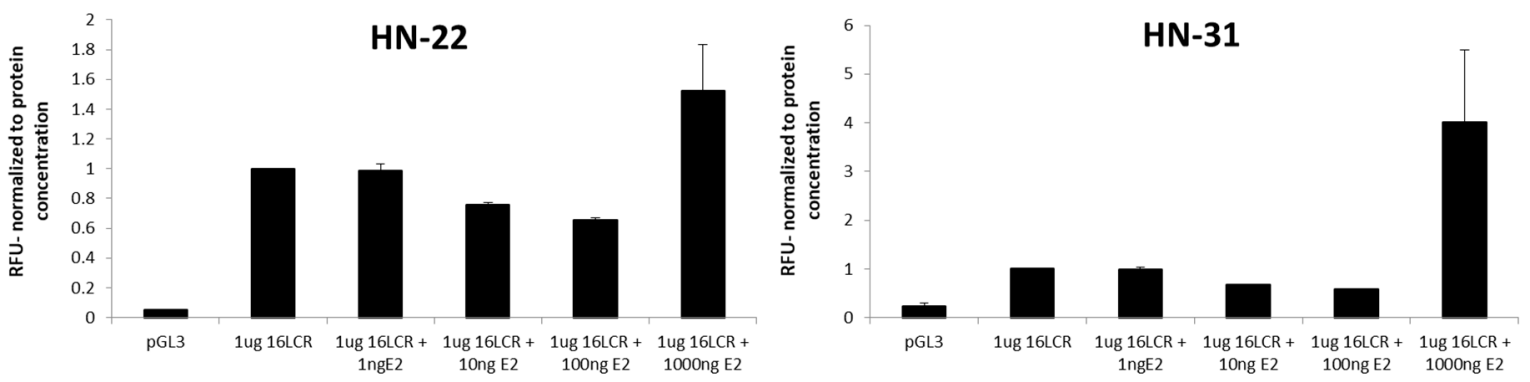

Figure 2. E2-mediated transcriptional activation and repression in $\mathrm{HN}-22$ and $\mathrm{HN}-31 \mathrm{HNSCC}$ cells. (A) Luciferase transcription activation assay in HN-22 and HN-31 utilizing pTK6E2. Relative luciferase units increase with increasing amounts of E2 protein present, indicating E2 mediated transcriptional activation of pTK6E2. pGL3 and pTK6E2 (no E2) lanes are shown as controls. (B) Luciferase transcription repression assay in HN-22 and HN-31 utilizing pGL3-16LCR (16LCR). Relative luciferase units decrease upon addition of E2 indicating transcriptional repression. At 1000ng E2 transcription is increased past baseline. pGL3 is shown as a negative control while pGL3-16LCR (no E2) represents basal, unrepressed transcription levels. ( $n=3$, error bars represent 


\subsection{Endogenous levels of IFIT1 determine the viability of E1-E2 mediated DNA replication in HNSCC cells}

While HN-22 and HN-31 cells allowed for E1-E2 mediated DNA replication and for E2-mediated transcriptional activation and repression, the other transfectable HNSCC lines tested did not allow for detectable DNA replication when transfected with pOri+E1+E2. Others have previously shown that the innate immune response protein, IFIT1, binds the HPV18 E1 protein and inhibits HPV18 replication [51, 52]. In many cell types, IFIT1 is expressed at low levels and is induced in response by interferon in the presence of pathogens [53]. Our previous work in oral keratinocytes demonstrated that NOKs (TERT-immortalized normal oral keratinocytes) express high levels of endogenous IFIT1, which is greatly reduced upon introduction of the HPV16 genome [54]. We hypothesized that IFIT1 may also bind the HPV16 E1 protein and that high levels of endogenous IFIT1 in certain HNSCC cell lines may explain the discrepancy in E1-E2 mediated replication observed among the HNSCC cells tested. Western blotting for IFIT1 in HNSCC cell lines revealed that HN-22 and HN-31 lines had no detectable endogenous IFIT1 protein (Figure 3). All other cells tested, with detectable IFIT1 via western blot analysis, did not allow for HPV16 E1-E2 mediated DNA replication. HN-8 cells also had low levels of IFIT1 but had poor transfectability (Table 1) and thus could not be used for our replication assay. This result suggests that higher levels of endogenous IFIT1 may interfere with HPV16 E1-E2 mediated DNA replication in HNSCC cells.

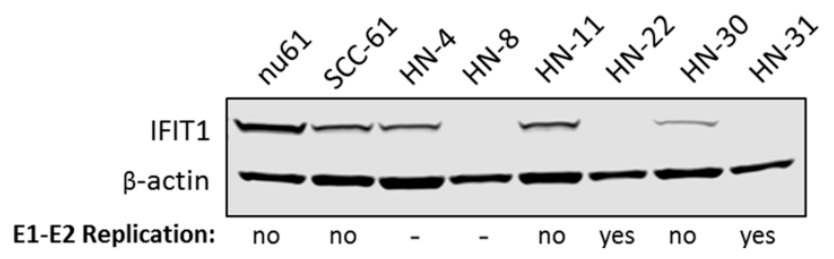

\footnotetext{
Figure 3. Low levels of IFIT1 are required for E1-E2 replication. Western blot analysis demonstrating levels of endogenous IFIT1 in HNSCC cell lines. $\beta$-actin is shown as a loading control. Of cell lines that were transfectable, only HN-22 and HN-31 (no detectable IFIT1) allowed for robust, measurable E1-E2 mediated DNA replication.
}

3.5 Exogenous IFIT1 binds HPV16 E1 in HN-22 cells and significantly attenuates E1-E2 mediated DNA replication

In order to determine if the mechanism of IFIT1 replication restriction in HNSCC cells was similar to that reported in HPV18 in HT-1080 fibrosarcoma cells [52], HN-22 cells were transfected with varying combinations of pOri, HPV16 E1, HPV16 E2, and FLAG-tagged IFIT1. Input blots indicated robust expression of E1, E2 and IFIT1 after transfection (Figure 4). Interestingly, E1 appears to be stabilized in the presence of IFIT1 (input lane 2) but not when E2 is also present (input lane 4). When an anti-FLAG antibody was used to immunoprecipitate FLAG-IFIT1 associated proteins, HPV16 E1 was demonstrated to interact with IFIT1 (IP lane 2). This is the first report of an interaction between HPV16 E1 and IFIT1, as well as the first report of any interaction between E1 and IFIT1 in HNSCC cells. Interestingly, this interaction appears to be abrogated in the presence of the HPV16 E2 protein, suggesting there may be competition between E2 and IFIT1 for E1 binding (IP lane 4). 


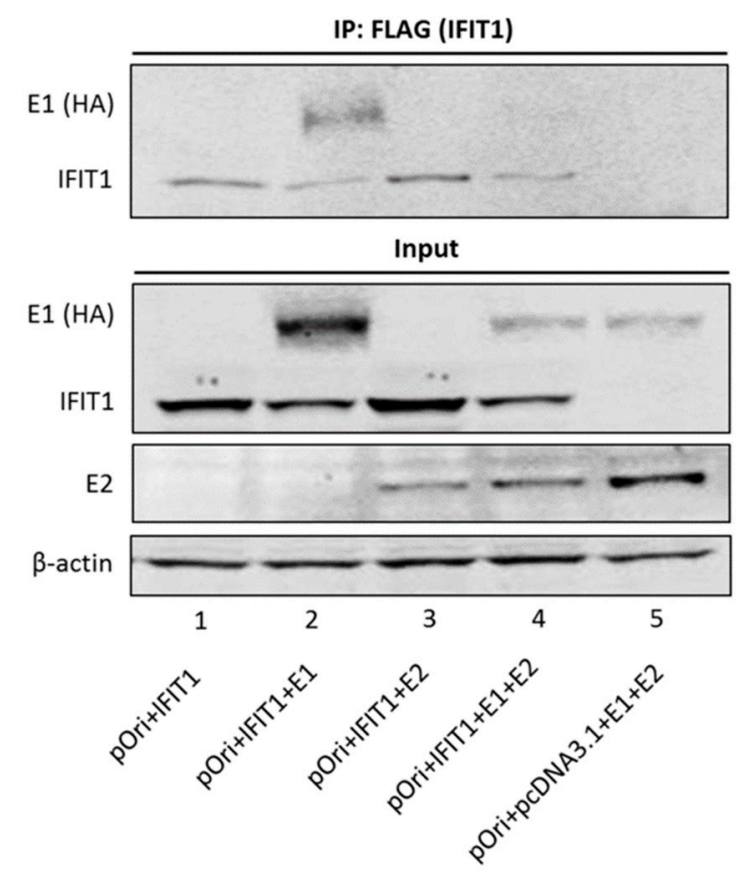

Figure 4. IFIT1 binds HPV16 E1 in HN-22 cells. Immunoprecipitation experiment demonstrating an interaction between HPV16 E1 and exogenous FLAG-IFIT1 in HN-22 cells (IP lane 2). This interaction is abrogated in the presence of E2 (IP lane 4). $\beta$-actin is shown as a loading control for the input blot.

303 To determine if addition of exogenous IFIT1 attenuated E1-E2 mediated DNA replication in 304 HNSCC cells, pOri, E1, E2, were cotransfected into HN-22 cells as performed in Figure 1. To directly compare levels of E1-E2 mediated DNA replication, HN-22 cells were also transfected with pOri+E1+E2+IFIT1. HN-22 cells transfected with IFIT1 alongside the complete replication complex (pOri+E1+E2) demonstrated a trend towards less E1-E2 mediated replication compared to pOri+E1+E2 cells but the effect was variable in magnitude (Figure $5 \mathrm{~A}$ ). These results suggested there may be some temporality to the interaction between IFIT1 and E1. Because IFIT1 is primarily cytoplasmic, IFIT1 may need to be expressed before E1 is translocated to the nucleus in order to bind E1 and attenuate E1-E2 mediated replication. To test this, HN-22 cells were cotransfected with IFIT1 and one of two resistance plasmids (pLX302-puromycin or pBABE-hygromycin) and selected with the appropriate antibiotic to create two cell lines stably overexpressing IFIT1 (HN-22+IFIT1-puro and HN-22+IFIT1-hygro). Increased levels of IFIT1 are observed in HN-22+IFIT1-puro and HN-22+IFIT1-hygro lines compared to the parental HN22 cell line (Figure 5 B). To determine if overexpression of IFIT1 in these lines attenuated replication, HN-22+IFIT1-puro and HN-22+IFIT1-hygro lines were transfected with pOri, pOri+E1 or pOri+E1+E2 alongside the parental HN22 line. As expected, only cells transfected with the full replication complex (pOri+E1+E2) demonstrated significant amounts of replication. Significantly less replication was observed in HN-22+IFIT1-puro and HN-22+IFIT1-hygro transfected with the full replication complex compared to the parental HN-22 line (Figure $5 \mathrm{C}$ ). These results suggest that high levels of IFIT1, present before the expression of HPV proteins E1 and E2, leads to less E1-E2 mediated DNA replication. This reduction of replication in HN-22+IFIT1 lines is presumably due to the previously described interactions between IFIT1 and E1 (Figure 4). 
C

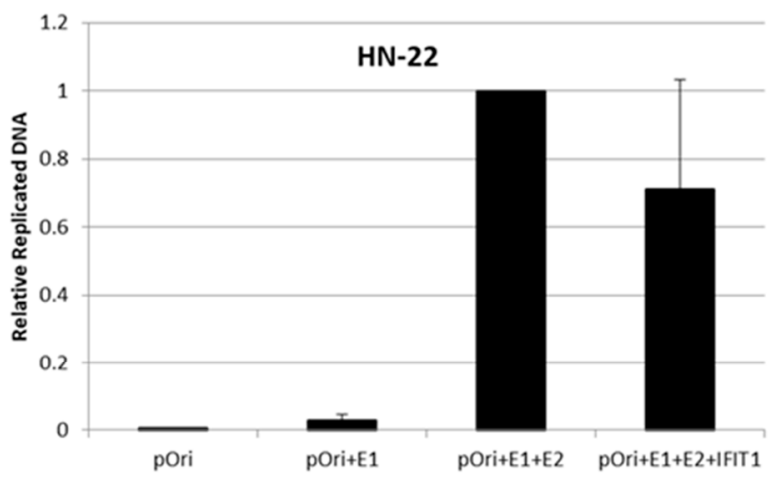

B

C

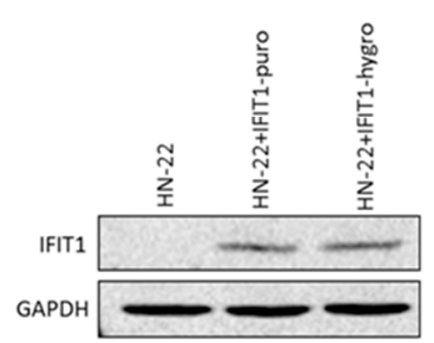
HN-22+IFIT1-hygro ( $\mathrm{p}=0.010)$.

\section{Discussion}

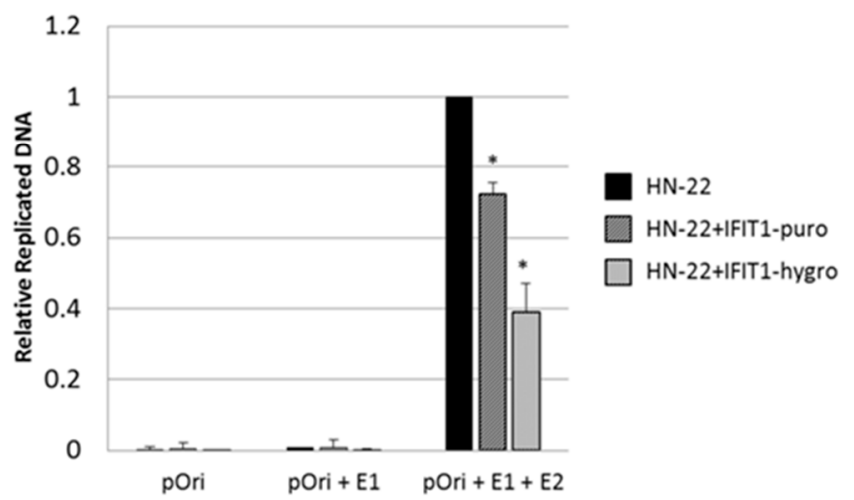

Figure 5. IFIT1 attenuates HPV E1-E2 mediated DNA replication. (A) HN-22 cells were transfected with varying combinations of $1 \mu \mathrm{g}$ IFIT1, $1 \mu \mathrm{g}$ pOri, $1 \mu \mathrm{g}$ E1 and 10ng E2 and E1-E2 mediated replication was determined by real-time PCR for pOri. Cells expressing pOri+E1+E2+IFIT1 displayed a nonsignificant trend toward less replication than cells transfected with pOri+E1+E2. $(n=3$, error bars represent SEM). (B) Western blot analysis demonstrating increased expression of IFIT1 in HN-22+IFIT1 cell lines compared to the HN-22 parental line. GAPDH is shown as a loading control. (C) HN-22, HN-22+IFIT1-puro, and HN-22+IFIT1-hygro were transfected with varying combinations of $1 \mu \mathrm{g}$ pOri, $1 \mu \mathrm{g}$ E1 and 10ng E2 and E1-E2 mediated replication was determined by real-time PCR for pOri. Significant replication was observed only in cells transfected with the full HPV16 replication complex (pOri+E1+E2) $(n=3$, error bars represent SEM). Compared to the parental HN-22 line, significantly less replication was observed in HN-22+IFIT1-puro ( $\mathrm{p}=0.006)$ and

Although prophylactic vaccines are available to prevent future HPV16 infections, there are currently no specific anti-HPV treatments for HPV+HNSCC, of which $90 \%$ are attributable to HPV16 [3]. Contrary to other HPV+ cancers, in a majority of HPV+HNSCCs, the HPV genome remains episomal and is replicated in a E1-E2 dependent manner [23-26]. In light of the differences in genome structure of HPV+HNSCC compared to other HPV+ cancers, as well as cell-specific differences in transcriptional control of viral genes observed in other HPV types [55], we set out to create a model system to reproducibly study HPV16 E1-E2 mediated replication and E2-mediated transcription in HNSCC. Our results demonstrate that expression of a complete HPV16 replication complex (pOri+E1+E2) in HN-22 and HN-31 leads to robust, reproducible E1-E2 mediated replication as well as replication foci formation (Figure 1). HN-22 and HN-31 also demonstrate E2-mediated transcriptional activation of pTK6E2 that is dose dependent (Figure 2A). Interestingly, E2-mediated repression of pGL3-16LCR transcription in HN-22 and HN-31 appears dose dependent 
up to a certain level of E2, however this repression appears to be lost when higher amounts of E2 (1000ng) are expressed alongside pGL3-16LCR (Figure 2 B). This phenomenon is not observed in cells from other anatomical regions [56-59] and highlights the potential for cell-type or tissue-specific differences in HPV16 transcription in HNSCC. Overall, our model is well-suited to study and better characterize E2-mediated transcription and E1-E2 mediated replication in HNSCC.

In developing our real-time PCR assay to measure E1-E2 mediated replication in HNSCC cells, we discovered that low endogenous levels of IFIT1 are required for measurable replication to occur (Figure 3). This is supported by our previous findings that IFIT1 is significantly downregulated by HPV16 in oral keratinocytes [54]. IFIT1 is an interferon induced protein that exerts numerous known antiviral functions [53]. IFIT1 has been shown to have nonspecific antiviral functions, such as binding eukaryotic initiation factor 3 (eIF3) and inhibiting cellular and viral translation, as well as by binding directly viral RNAs that lack 2'-O-methylation [60, 61]. Because HPVs utilize cellular transcriptional machinery, HPV transcripts should contain 2'-O-methylation, so this latter mechanism is likely not relevant in HPV infections. However, specifically relating to HPV, IFIT1 has been shown to bind the HPV18 E1 protein in 1080T fibrosarcoma cells [51]. We observe a similar binding interaction between HPV16 E1 and IFIT1 in HN-22 cells (Figure 4) and hypothesize that this E1-IFIT1 interaction is the primary anti-HPV action of IFIT1. This is supported by our results demonstrating that overexpression of IFIT1 in HN-22 cells leads to less E1-E2 mediated replication than in parental HN-22 cells that express no detectable IFIT1 (Figure 5 C).

Our results demonstrate that the effect of IFIT1 on HPV E1-E2 mediated replication in our system is more significant when IFIT1 is expressed before transfection with E1, E2, and pOri. It is possible that the role of IFIT1 in restricting HPV replication is most important during the initial establishment stage of the HPV life cycle. Cells with high endogenous IFIT1 expression may be more resistant to initial HPV infection, giving possible insight as to why not all HPV infections result persistent disease. Further studies are necessary to determine if there is such temporality to the IFIT1-E1 interaction or if it is simply a matter of IFIT1 levels being sufficient to out-compete E2 for E1 binding, as the presence of E2 appears to reduce the IFIT1-E1 interaction (Figure 4). It is of note that overexpression of IFIT1 in HN-22 only reduces replication by about 50\% (Figure 5 C). There are likely other factors that negatively regulate HPV replication, as numerous innate immune factors like IFIT1, have been shown to be downregulated by HPV16 in cells of the head and neck region and in HPV+HNSCC clinical samples [54, 62]. While further characterization of the HPV16 IFIT1-E1 interaction should be investigated to inform future anti-viral therapies, there is reason to believe that other interactions, pertaining to both HPV16 replication and transcription, can be discovered and tested utilizing this HNSCC model system. Overall, this model system can be used to study HPV16 E1-E2 mediated replication and E2-dependent transcription in HNSCC cells and could be used as a tool to screen replication and transcription targeting anti-HPV agents. 
393

394

395

396

397

398

399

400

401

402

Acknowledgments: I.M.M received funding from the Virginia Commonwealth University Philips Institute for Oral Health Research and the Virginia Commonwealth University National Cancer Institute Designated Massey Cancer Center, NIH P30 CA016059. Microscopy was performed at the VCU Microscopy Facility, supported in part, by funding from National Institutes of Health-National Cancer Institute Cancer Center Support Grant P30 CA016059.

Author Contributions: M.R.E., M.L.B. and I.M.M conceived and designed experiments. M.R.E., C.T.F., M.L.B. performed and analyzed most experiments. C.D.J. and M.L.B. performed immunofluorescence experiments. C.D.J. performed cloning. X.W. performed real-time PCR. M.R.E. wrote the manuscript and C.D.J. edited the manuscript.

Conflicts of Interest: The authors declare no conflict of interest. 
403

404

405

406

407

408

409

410

411

412

413

414

415

416

417

418

419

420

421

422

423

424

425

426

427

428

429

430

431

432

433

434

435

436

437

438

439

440

441

442

\section{References}

1. zur Hausen, H. Papillomaviruses in the Causation of Human Cancers - a Brief Historical Account. Virology. 2009, 384, 260-265.

2. Chesson, H.W.; Dunne, E.F.; Hariri, S.; Markowitz, L.E. The Estimated Lifetime Probability of Acquiring Human Papillomavirus in the United States. Sex. Transm. Dis. 2014, 41, 660-664.

3. Psyrri, A.; DiMaio, D. Human Papillomavirus in Cervical and Head-and-Neck Cancer. Nat. Clin. Pract. Oncol. 2008, 5, 24-31.

4. Marur, S.; D'Souza, G.; Westra, W.H.; Forastiere, A.A. HPV-Associated Head and Neck Cancer: A Virus-Related Cancer Epidemic. Lancet Oncol. 2010, 11, 781-789.

5. Walker, T.Y.; Elam-Evans, L.D.; Yankey, D.; et al. National, Regional, State, and Selected Local Area Vaccination Coverage Among Adolescents Aged 13-17 Years - United States, 2017. MMWR Morb Mortal Wkly Rep. 2018, 67, 909-917.

6. Munger, K.; Phelps, W.C.; Bubb, V.; Howley, P.M.; Schlegel, R. The E6 and E7 Genes of the Human Papillomavirus Type 16 Together are Necessary and Sufficient for Transformation of Primary Human Keratinocytes. J. Virol. 1989, 63, 4417-4421.

7. Munger, K.; Werness, B.A.; Dyson, N.; Phelps, W.C.; Harlow, E.; Howley, P.M. Complex formation of human papillomavirus E7 proteins with the retinoblastoma tumor suppressor gene product. EMBO J. 1989, 8,4099-4105.

8. Dyson, N.; Howley, P.M.; Munger, K.; Harlow, E. The human papillomavirus-16 E7 oncoprotein is able to bind to the retinoblastoma gene product. Science. 1989, 243, 934-937.

9. Scheffner, M.; Werness, B.A.; Huibregtse, J.M.; Levine, A.J.; Howley, P.M. The E6 oncoprotein encoded by human papillomavirus types 16 and 18 promotes the degradation of p53. Cell. 1990, 63, $1129-1136$.

10.Sedman, S.A.; Barbosa, M.S.; Vass, W.C.; Hubbert, N.L.; Haas, J.A.; Lowy, D.R.; Schiller, J.T. The Full-Length E6 Protein of Human Papillomavirus Type 16 has Transforming and Trans-Activating Activities and Cooperates with E7 to Immortalize Keratinocytes in Culture. J. Virol. 1991, 65, 4860-4866.

11.Werness, B.A.; Munger, K.; Howley, P.M.. Role of the human papillomavirus oncoproteins in transformation and carcinogenic progression. Important Adv. Oncol. 1991, 3-18. 
12.Huibregtse, J.M.; Scheffner, M.; Howley, P.M. Cloning and expression of the cDNA for E6AP, a protein that mediates the interaction of human papillomavirus E6 oncoprotein with p53. Molecular and Cellular Biology. 1993, 13, 775-784.

13. Riley, R.R.; Duensing, S.; Brake, T.; Munger, K.; Lambert, P.F.; Arbeit, J.M. Dissection of Human Papillomavirus E6 and E7 Function in Transgenic Mouse Models of Cervical Carcinogenesis. Cancer Res. 2003, 63, 4862-4871.

14.Huh, K.; Zhou, X; Hayakawa, H.; Cho, J.Y.; Libermann, T.A.; Jin, J.; Harper, J.W.; Munger, K. Human papillomavirus type 16 E7 oncoprotein associates with the cullin 2 ubiquitin ligase complex, which contributes to degradation of the retinoblastoma tumor suppressor. Journal of Virology. 2007, 81, 9737-9747.

15.Jiang, M.; Milner, J. Selective silencing of viral gene expression in HPV-positive human cervical carcinoma cells treated with siRNA, a primer of RNA interference. Oncogene. 2002, 21, 604-6048.

16. Butz, K.; Ristriani, T.; Hengstermann, A.; Denk, C.; Scheffner, M.; Hoppe-Seyler, F. SiRNA Targeting of the Viral E6 Oncogene Efficiently Kills Human Papillomavirus-Positive Cancer Cells. Oncogene. 2003, 22, 5938-5945.

17.Hall, A.H.; Alexander, K.A. RNA Interference of Human Papillomavirus Type 18 E6 and E7 Induces Senescence in HeLa Cells. J. Virol. 2003, 77, 6066-6069.

18. Yoshinouchi, M.; Yamada, T.; Kizaki, M.; Fen, J.; Koseki, T.; Ikeda, Y.; et al. In vitro and in vivo growth suppression of human papillomavirus16-positive cervical cancer cells by E6 siRNA. Mol Ther. 2003, 8, 762-768.

19.Gu, W.; Putral, L.; Hengst, K.; Minto, K.; Saunders, N.A. Leggatt, G.; et al. Inhibition of cervical cancer cell growth in vitro and in vivo with lentiviral-vector delivered short hairpin RNA targeting human papillomavirus E6 and E7 oncogenes. Cancer Gene Ther. 2006, 13, 1023-1032.

20. Yamato, K.; Yamada, T.; Kizaki, M.; Ui-Tei. K.; Natori, Y.; Fujino, M.; Nishihara, T.; Ikeda, Y.; Nasu, Y.; Saigo, K.; Yoshinouchi, M. New highly potent and specific E6 and E7 siRNAs for treatment of HPV16 positive cervical cancer. Cancer Gene Therapy. 2008, 15, 140-153.

21.Jabbar, S.F.; Abrams, L.; Glick, A.; Lambert, P.F. Persistence of high-grade cervical dysplasia and cervical cancer requires the continuous expression of the human papillomavirus type $16 \mathrm{E} 7$ oncogene. Cancer Research. 2009, 69, 4407-4414

22.Hoppe-Seyler, K.; Bossler, F; Braun, J.A.; Herrmann, A.L.; Hoppe-Seyler, F. The HPV E6/E7 Oncogenes: Key Factors for Viral Carcinogenesis and Therapeutic Targets. Trends in Microbiology. 2018, 26, 158-168. 
23.Parfenov, M.; Pedamallu, C.S.; Gehlenborg, N.; Freeman, S.S.; Danilova, L.; Bristow, C.A.; Lee, S.; Hadjipanayis, A.G.; Ivanova, E.V.; Wilkerson, M.D.; et al. Characterization of HPV and Host Genome Interactions in Primary Head and Neck Cancers. Proc. Natl. Acad. Sci. U. S. A. 2014, 111, 15544-15549.

24. Cancer Genome Atlas Network. Comprehensive Genomic Characterization of Head and Neck Squamous Cell Carcinomas. Nature. 2015, 517, 576-582.

25. Ramqvist, T.; Mints, M.; Tertipis, N.; Nasman, A.; Romanitan, M.; Dalianis, T. Studies on Human Papillomavirus (HPV) 16 E2, E5 and E7 mRNA in HPV-Positive Tonsillar and Base of Tongue Cancer in Relation to Clinical Outcome and Immunological Parameters. Oral Oncol. 2015, 51, $1126-1131$.

26. Nulton, T.J.; Olex, A.L.; Dozmorov, M.; Morgan, I.M.; Windle, B. Analysis of the Cancer Genome Atlas Sequencing Data Reveals Novel Properties of the Human Papillomavirus 16 Genome in Head and Neck Squamous Cell Carcinoma. Oncotarget. 2017, 8, 17684-17699.

27.Steger, G.; Corbach, S. Dose-dependent regulation of the early promoter of human papillomavirus type 18 by the viral E2 protein. J. Virol. 1997, 71, 50-58.

28. Desaintes, C.; Demeret, C.; Goyat, S.; Yaniv, M.; Thierry, F. Expression of the papillomavirus E2 protein in HeLa cells leads to apoptosis. EMBO J. 1997, 16, 504-514.

29. Goodwin, E.C.; Naeger, L. K.; Breiding, D. E.; Androphy, E. J.; DiMaio, D.

Transactivation-competent bovine papillomavirus E2 protein is specifically required for efficient repression of human papillomavirus oncogene expression and for acute growth inhibition of cervical carcinoma cell lines. J. Virol. 1998, 72, 3925-3934.

30.Goodwin, E.C.; Yang, E.; Lee, C.J.; Lee, H.W.; DiMaio, D.; Hwang, E.S. Rapid induction of senescence in human cervical carcinoma cells. Proc. Natl. Acad. Sci. USA. 2000, 97, 10978-10983.

31.Francis, D.A.; Schmid, S.I.; Howley, P.M. Repression of the integrated papillomavirus E6/E7 promoter is required for growth suppression of cervical cancer cells. J. Virol. 2000, 74, 2679-2686.

32. Cardinali, M.; Pietraszkiewicz, H.; Ensley, J.F.; Robbins, K.C. Tyrosine phosphorylation as a marker for aberrantly regulated growth-promoting pathways in cell lines derived from head and neck malignancies. International Journal of Cancer. 1995, 61, 98-103.

33. Khodarev, N.N.; Beckett, M.; Labay, E.; Darga, T.; Roizman, B.; Weichselbaum, R.R. STAT1 is overexpressed in tumors selected for radioresistance and confers protection from radiation in transduced sensitive cells. Proc Natl Acad Sci USA. 2004, 101, 1714-1719.

34.Taylor, E.R.; Morgan, I.M. A Novel Technique with Enhanced Detection and Quantitation of HPV-16 E1- and E2-Mediated DNA Replication. Virology. 2003, 315, 103-109.

35. Kadaja, M.; Sumerina, A.; Verst, T.; Ojarand, M.; Ustav, E.; Ustav, M. Genomic Instability of the Host Cell Induced by the Human Papillomavirus Replication Machinery. EMBO J. 2007, 26, 2180-2191.

36. Bouvard, V.; Storey, A.; Pim, D.; Banks, L. Characterization of the Human Papillomavirus E2 Protein: Evidence of Trans-Activation and Trans-Repression in Cervical Keratinocytes. EMBO J. 1994, 13, 5451-5459. 
539

540

541

542

543

544

545

546

547

548

549

550

551

552

553

554

555

556

557

558

559

560

561

562

563

564

565

566

567

568

569

570

571

572

573

574

575

576

577

578

579

580

581

582

583

584

585

586

587

588

589
37.Katibah, G.E.; Lee, H.J.; Huizar, J.P.; Vogan, J.M.; Alber, T.; Collins, K. TRNA Binding, Structure, and Localization of the Human Interferon-Induced Protein IFIT5. Mol. Cell. 2013, 49, 743-750.

38. Yang, X.; Boehm, J.S.; Yang, X.; Salehi-Ashtiani, K.; Hao, T.; Shen, Y.; Lubonja, R.; Thomas, S.R.; Alkan, O.; Bhimdi, T.; Green, T.M.; Johannessen, C.M.; Silver, S.J.; Nguyen, C.; Murray, R.R.; Hieronymus, H.; Balcha, D.; Fan, C.; Lin, C.; Ghamsari, L.; Vidal, M.; Hahn, W.C.; Hill, D.E.; Root, D.E. A public genome-scale lentiviral expression library of human ORFs. Nat Methods. 2011, 8, 659-661.

39. Morganstern, J.P.; Land, H. Advanced mammalian gene transfer: high titre retroviral vectors with multiple drug selection markers and a complementary helper-free packaging cell line. Nucleic Acids Res. 1990, 18, 3587-3596.

40.Vance, K.W.; Campo, M.S.; Morgan, I.M. An enhanced epithelial response of a papillomavirus promoter to transcriptional activators. J. Biol. Chem. 1999, 274, 27839-27844.

41. Bristol, M.L.; Wang, X.; Smith, N.W.; Son, M.P.; Evans, M.R.; Morgan, I.M. DNA Damage Reduces the Quality, but Not the Quantity of Human Papillomavirus 16 E1 and E2 DNA Replication. Viruses 2016, 8, 10.3390/v8060175.

42.Boner, W.; Taylor, E.R.; Tsirimonaki, E.; Yamane, K.; Campo, M.S.; Morgan, I.M. A Functional Interaction between the Human Papillomavirus 16 transcription/replication Factor E2 and the DNA Damage Response Protein TopBP1. J. Biol. Chem. 2002, 277, 22297-22303.

43. Kingston, R.E.; Chen, C.A.; Rose, J.K. Calcium Phosphate Transfection. Curr. Protoc. Mol. Biol. 2003, Chapter 9, Unit 9.1.

44.Swindle, C.S.; Zou, N.; Van Tine, B.A.; Shaw, G.M.; Engler, J.A.; Chow, L.T. Human Papillomavirus DNA Replication Compartments in a Transient DNA Replication System. J. Virol. 1999, 73, 1001-1009.

45. Tan, S.H.; Gloss, B.; Bernard H.U. During negative regulation of the human papillomavirus-16E6 promoter, the viral E2 protein can displace Sp1 from a proximal promoter element. Nucleic Acids Res. 1992, 20, 251-256.

46. Tan, S.H.; Leong, L.E.; Walker, P.A.; Bernard, H.U. The human papillomavirus type 16 E2 transcription factor binds with low cooperativity to two flanking sites and represses the E6 promoter through displacement of Sp1 and TFIID. J. Virol. 1994, 68, 6411-6420.

47.Dong, G.; Broker, T.R.; Chow, L.T. Human papillomavirus type 11 E2 proteins repress the homologous E6 promoter by interfering with the binding of host transcription factors to adjacent elements. J. Virol. 1994, 68, 1115-1127.

48.Soeda, E.; Ferrari, M.C.; Baker, C.C.; McBride, A.A. Repression of HPV16 early region transcription by the E2 protein. Virology. 2006, 351, 29-41.

49.Smith, J.A.; Haberstroh, F.S.; White, E.A.; Livingston, D.M.; DeCaprio, J.A., Howley, P.M. SMCX and components of the TIP60 complex contribute to E2 regulation of the HPV E6/E7 promoter. Virology. 2014, 468-470:311-321. 
590

591

592

593

594

595

596

597

598

599

600

601

602

603

604

605

606

607

608

609

610

611

612

613

614

615

616

617

618

619

620

621

622

623

624

625

626

627

628

629

630

631

632

633

634

635

636

50.Gauson, E.J.; Donaldson, M.M.; Dornan. E.S.; Wang, X.; Bristol, M.L.; Bodily, J.M.; Morgan, I.M. Evidence supporting a role for TopBP1 and Brd4 in the initiation but not continuation of human papillomavirus 16 E1/E2-mediated DNA replication. J Virol. 2015, 89, 4980-4991.

51.Terenzi, F.; Saikia, P.; Sen, G.C. Interferon-Inducible Protein, P56, Inhibits HPV DNA Replication by Binding to the Viral Protein E1. EMBO J. 2008, 27, 3311-3321.

52.Saikia, P.; Fensterl, V.; Sen, G.C. The Inhibitory Action of P56 on Select Functions of E1 Mediates Interferon's Effect on Human Papillomavirus DNA Replication. J. Virol. 2010, 84, 13036-13039.

53.Fensterl, V.; Sen, G.C. Interferon-Induced Ifit Proteins: Their Role in Viral Pathogenesis. J. Virol. 2015, 89, 2462-2468.

54.Evans, M.R.; James, C.D.; Loughran, O.; Nulton, T.J.; Wang, X.; Bristol, M.L.; Windle, B.; Morgan, I.M. An Oral Keratinocyte Life Cycle Model Identifies Novel Host Genome Regulation by Human Papillomavirus 16 Relevant to HPV Positive Head and Neck Cancer. Oncotarget 2017, 47, 81892-81909.

55.Rapp, B.; Pawellek, A.; Kraetzer, F.; Schaefer, M.; May, C.; Purdie, K.; Grassman, K.; Iftner, T. Cell-type-specific separate regulation of the E6 and E7 promoters of human papillomavirus type 6a by the viral transcription factor E2. J. Virol. 1997, 71, 6956-6966.

56. Thierry, F.; Yaniv, M. The BPV1 E2 trans-acting protein can be either a repressor or activator of the HPV18 regulatory region. EMBO J. 1987, 6, 3391-3397.

57. Bernard, B.A.; Bailly, C.; Lenoir, M.C.; Darmon, M.; Thierry, F.; Yaniv M. The human papillomavirus type 18 (HPV18) E2 gene product is a repressor of the HPV18 regulatory region in human keratinocytes. J. Virol. 1989, 63, 4317-4324

58. Stenlund, A.; Botchan, M.R. The E2 trans-activator can act as a repressor by interfering with a cellular transcription factor. Genes Dev. 1990, 4, 123-136.

59.Nishimura, A.; Ono, T.; Ishimoto, A.; Dowhanick, J.J.; Frizzell, M.A.; Howley, P.M.; Sakai, H. Mechanisms of human papillomavirus E2-mediated repression of viral oncogene expression and cervical cancer cell growth inhibition. J. Virol. 2000, 8, 3752-3760.

60.Hui, D.J.; Bhasker, C.R.; Merrick, W.C.; Sen, G.C. Viral Stress-Inducible Protein p56 Inhibits Translation by Blocking the Interaction of eIF3 with the Ternary Complex eIF2.GTP.Met-tRNAi. J. Biol. Chem. 2003, 278, 39477-39482.

61.Habjan, M.; Hubel, P.; Lacerda, L.; Benda, C.; Holze, C.; Eberl, C.H.; Mann, A.; Kindler, E.; Gil-Cruz, C.; Ziebuhr, J.; et al. Sequestration by IFIT1 Impairs Translation of 2'O-Unmethylated Capped RNA. PLoS Pathog. 2013, 9, e1003663.

62. Evans, M.R.; James, C.D.; Bristol, M.L.; Wang, X.; Kaur, N.; White, E.A.; Windle, B.; Morgan, I.M. Human papillomavirus 16 E2 regulates keratinocyte gene expression relevant to cancer and the viral life cycle. J. Virol. 2018, doi: 10.1128/JVI.01941-18. 